\title{
Polyamines as modulators of microcycle conidiation in Aspergillus flavus
}

\author{
N. Khurana, ${ }^{1}$ R. K. Saxena, ${ }^{1}$ Rani Gupta ${ }^{1}$ and M. V. Rajam ${ }^{2}$ \\ Author for correspondence: M. V. Rajam. Tel: +91 11 606392. Fax: +91 $116886427 / 6885270$. \\ e-mail: bic_dusc@dbt.ernet.in
}

Departments of Microbiology ${ }^{1}$, and Genetics', University of Delhi, South Campus, Benito Juarez Road, New Delhi 110021, India

\begin{abstract}
Since polyamines (PAs) play a potential role in the regulation of growth and developmental processes in a wide variety of organisms, we have examined the influence of the PAs putrescine (Put) and spermidine (Spd) and the PA biosynthetic inhibitors $\alpha$-difluoromethylornithine (DFMO), $\alpha$ difluoromethylarginine (DFMA), methylglyoxal bis-(guanylhydrazone) (MGBG) and cyclohexylamine (CHA), singly and in combinations on microcycle conidiation (MC) in Aspergillus flavus. The exogenous application of the diamine Put (concentrations ranging from 0.1 to $5 \mathrm{mM}$ ) caused a sharp decline of MC in a dose-dependent fashion, but induced vegetative growth. However, the triamine Spd (0.1-5 mM) had a minimal effect on MC and induced a shift from MC to normal conidiation. PA inhibitors, especially DFMO, MGBG and CHA, produced greater inhibition of MC and complete inhibition of MC was observed at $5 \mathrm{mM}$ of these inhibitors. DFMA even at $5 \mathrm{mM}$ had only a weak inhibitory effect on MC. DFMO also inhibited conidial germination and germ tube growth. MGBG and CHA, while having an inhibitory effect on MC, induced vegetative growth. The inhibitory effect of PA inhibitors was partially reversed by exogenous Put or Spd, with Spd being more effective than Put. The analysis of free PA levels during various phases of MC revealed that undifferentiated spores contained a high Put/Spd ratio and there was a dramatic decrease in Put/Spd ratio before and during microcycle conidiophore maturity. The change in spermine titres could not be detected. These observations imply that Put is essential for vegetative growth, while Spd is involved in MC, and that a low Put/Spd ratio seems to be important for spore differentiation to MC.
\end{abstract}

Keywords: Aspergillus flavus, development, microcycle conidiation, polyamines, polyamine biosynthesis inhibitors

\section{INTRODUCTION}

Microcycle conidiation (MC) (a condensed developmental process of conidiation without any intervening vegetative mycelium) has been reported in Aspergillus niger using temperature manipulation by Anderson \& Smith $(1971 \mathrm{a}, \mathrm{b})$. Since then attempts have been made to induce this phenomenon in other Aspergillus spp. and fungi by manipulating culture conditions and/or nutrient factors (Maheshwari, 1991). Earlier, we reported MC on a novel medium, D-glucose soluble starch (D-GSS) in Aspergillus spp. (Saxena et al., 1992) and under light-independent

Abbreviations: CHA, cyclohexylamine; DFMA, $\alpha$-difluoromethylarginine; DFMO, $\alpha$-difluoromethylornithine; D-GSS, D-glucose soluble starch; MC, microcycle conidiation; MGBG, methylglyoxal bis-(guanylhydrazone); PA(s), polyamine(s); Put, putrescine; Spd, spermidine; Spm, spermine. conditions in Trichoderma spp. (Khurana et al., 1993). Although some work has been done on the induction and on the ultrastructure, the internal events regulating microcycle differentiation are still not fully understood (Maheshwari, 1991). However, our work on MC in DGSS medium implied that the osmolarity of medium can regulate $\mathrm{MC}$, at the transcriptional level (Khurana et al., 1992).

Polyamines (PAs), spermidine (Spd) and spermine (Spm) and their precursor the diamine putrescine (Put) are involved in a variety of growth and developmental events in a wide range of organisms, including fungi (Rajam \& Galston, 1985; Rajam et al., 1985, 1986, 1989; Tabor \& Tabor, 1985; Walters, 1987; Singhania, 1991; Rajam, 1993). No attempts so far have been made to demonstrate the possible role of PAs in MC. Thus, we have examined the influence of exogenous PAs Put and Spd, and specific 
PA biosynthesis inhibitors $\alpha$-difluoromethylornithine (DFMO, a potent inhibitor of ornithine decarboxylase), $\alpha$-difluoromethylarginine (DFMA, a potent inhibitor of arginine decarboxylase), methylglyoxal bis-(guanylhydrazone) (MGBG, an inhibitor of adenosylmethionine decarboxylase, an enzyme which furnishes aminopropyl groups to Put for the formation of Spd and Spm) and cyclohexylamine (CHA, an inhibitor of Spd synthase) on MC in $A$. flavus. We have also measured the temporal changes in intracellular PA levels during various phases of MC.

\section{METHODS}

Growth conditions. A. flavus was maintained on a complete medium (Saxena \& Sinha, 1973; Saxena, 1976) slants at $4{ }^{\circ} \mathrm{C}$. For initiation of liquid cultures, conidia were harvested from 3d-old agar cultures using normal saline and $0 \cdot 01 \%$ Tween-80 as a detergent. Conidial harvest was filtered through sterilized cotton, centrifuged for $15 \mathrm{~min}$ at $1000 \mathrm{~g}$, washed twice with sterilized water, and resuspended in normal saline before being dispensed in culture flasks.

The normal developmental pattern was investigated in minimal medium (Saxena \& Sinha, 1973; Saxena, 1976). Liquid shake cultures were grown in $50 \mathrm{ml}$ medium in $250 \mathrm{ml}$ Erlenmeyer flasks, seeded with about $1 \times 10^{7}$ conidia. Flasks were incubated at $37^{\circ} \mathrm{C}$ at 250 r.p.m. in an incubation shaker (New Brunswick Scientific). Microscopic observations were recorded after a regular period of $4 \mathrm{~h}$.

Induction of MC. MC was induced by using a new medium DGSS previously described by Saxena et al. (1992). The medium contained $5 \mathrm{~g}$ D-glucose $\mathrm{l}^{-1}, 3.5 \mathrm{~g}$ soluble starch $\mathrm{l}^{-1}$ and $130 \mathrm{mg}$ $\mathrm{KH}_{2} \mathrm{PO}_{4} 1^{-1}$. Approximately $5 \times 10^{7}$ conidia were inoculated in $250 \mathrm{ml}$ Erlenmeyer flasks containing D-GSS medium. Flasks were incubated in an incubation shaker at $37^{\circ} \mathrm{C}$ and 250 r.p.m. MC was assessed by direct counting of conidia harvested at a regular period of $2 \mathrm{~h}$. Conidiophore counts included both immature and mature structures. MC was highly synchronous and about $99 \%$ conidia undergo MC in D-GSS medium.

PA extraction and quantification. Fungal spores and/or mycelial mats were obtained from liquid cultures grown at $37 \pm 1^{\circ} \mathrm{C}$ and 250 r.p.m. in $250 \mathrm{ml}$ Erlenmeyer flasks containing $50 \mathrm{ml}$ sterile D-GSS for various incubation times. Fungal biomass was allowed to air dry at room temperature and ground in a pre-chilled mortar with $5 \%(\mathrm{v} / \mathrm{v})$ pre-chilled $\mathrm{HClO}_{4}$ at a ratio of $1 \mathrm{ml} \mathrm{HClO}_{4}$ per $0 \cdot 1 \mathrm{~g}$ fresh weight. The homogenates were centrifuged at $15000 \mathrm{~g}$ for $20 \mathrm{~min}$ at $4{ }^{\circ} \mathrm{C}$. The free PAs in the supernatant fraction were dansylated along with standard PA mixture (Bajaj \& Rajam, 1995) and extracted in $250 \mu \mathrm{l}$ benzene, and the clear supernatant was used for the determiantion of PAs using high-resolution TLC plates (silica gel 60 of $250 \mu \mathrm{m}$ thickness; E. Merk, Germany). The TLC plates were developed in cyclohexane: ethylacetate $(5: 4, \mathrm{v} / \mathrm{v})$ solvent system. Dansylated PA bands marked under a UV lamp were scraped off, eluted in ethylacetate and quantified using a spectrophotofluorometer (Shimadzu RF540) with excitation and emission wavelengths at 350 and $495 \mathrm{~nm}$, respectively.

Effect of PAs and PA biosynthesis inhibitors, singly and in combinations on MC. Filter-sterilized stock solutions of Put and Spd and their biosynthesis inhibitors (DFMO, DFMA, MGBG and CHA), were added to autoclaved D-GSS liquid medium that had been cooled to room temperature, to get final concentrations of $0 \cdot 1,0 \cdot 5,1$ and $5 \mathrm{mM}$ of these compounds. For experiments involving reversal of inhibition, we utilized $5 \mathrm{mM}$
MGBG and $1 \mathrm{mM}$ DFMO inhibitor (which yielded $90-100 \%$ MC inhibition) with four concentrations $(0.01,0.1,0.5$ and $1.0 \mathrm{mM}$ ) of Put and Spd. Control flasks contained only culture medium with no PA/PA inhibitor. The cultures (in duplicate) containing conidia were incubated as described above. Microscopic observations were made after $24 \mathrm{~h}$ of incubation, which included both mature as well as immature conidiophore counts along with observation on vegetative and ungerminated conidia. The data were scored as percentage MC.

\section{RESULTS}

\section{Cellular PA levels during various phases of MC}

The free pools of PAs estimated during various stages of MC of A. flavus are given in Table 1 . The results show that all three common PAs were present, Put being predominant followed by Spd and Spm. Conidia and germinating spores contained a high Put/Spd ratio; however, as the germ tube committed to undergo MC (after $18 \mathrm{~h}$ of culture), there was a dramatic decrease in Put titres with a concomitant increase in Spd titres resulting in a reduction of Put/Spd ratio by about $50 \%$. The changes in Spm titre could not be detected.

\section{Effect of PAs on MC}

Conidia of $A$. flavus undergo a phase of vegetative growth (Fig. 2a) followed by normal asexual differentiation on $48 \mathrm{~h}$ of incubation in minimal medium (Fig. 1). However, on D-GSS medium, A. flavus shows four distinct developmental stages of $\mathrm{MC}$ : conidial germination stage which lasts for $2-3 \mathrm{~h}$ followed by germ-tube elongation (4-8 h); vesicle induction and phialodogenesis (10-16 h); and conidiogenesis (18-24 h; Fig. 2b) as shown in Fig. 1. On supplementation of D-GSS medium with various concentrations $(0.1,0.5$ and $1.0 \mathrm{mM})$ of Put or Spd, a progressive decline in $\mathrm{MC}$ was observed; Put being more inhibitory than Spd. Put caused a greater inhibition of MC even at a very low concentration of Put $(0.1 \mathrm{mM})$ where

\section{Table 1. Temporal changes in cellular free PA levels during various phases of $M C$}

PA levels [nmol $(\mathrm{g} \text { fresh } \mathrm{wt})^{-1}$ ] are means $\pm \mathrm{SD}$, based on two replicates. Experiment was repeated with similar results and the data presented are of one representative experiment. Spm titres did not change appreciably, hence they are not included in the table.

\begin{tabular}{|c|c|c|c|c|}
\hline $\begin{array}{l}\text { Age of } \\
\text { culture (h) }\end{array}$ & $\begin{array}{c}\text { Developmental } \\
\text { stage of } \mathbf{M C} *\end{array}$ & Put & Spd & $\begin{array}{c}\text { Put } / \text { Spd } \\
\text { ratio }\end{array}$ \\
\hline 0 & A & $631 \cdot 4 \pm 17 \cdot 2$ & $91 \cdot 5 \pm 12 \cdot 0$ & 6.90 \\
\hline 4 & B & $585.5 \pm 21.9$ & $82.5 \pm 3.5$ & $7 \cdot 09$ \\
\hline 8 & $\mathrm{C}$ & $709 \cdot 5 \pm 27 \cdot 6$ & $99.5 \pm 0.7$ & $7 \cdot 13$ \\
\hline 18 & $\mathrm{D}$ & $674.5 \pm 20.5$ & $172 \cdot 5 \pm 3 \cdot 5$ & $3 \cdot 91$ \\
\hline 24 & $\mathrm{E}$ & $464.5 \pm 21.9$ & $180 \cdot 8 \pm 2 \cdot 8$ & $2 \cdot 57$ \\
\hline
\end{tabular}

* Various developmental stages of MC: A, freshly inoculated conidia; B, germ-tube initiation; C, germ-tube elongation; D, philadogenesis; E, conidiogenesis. 


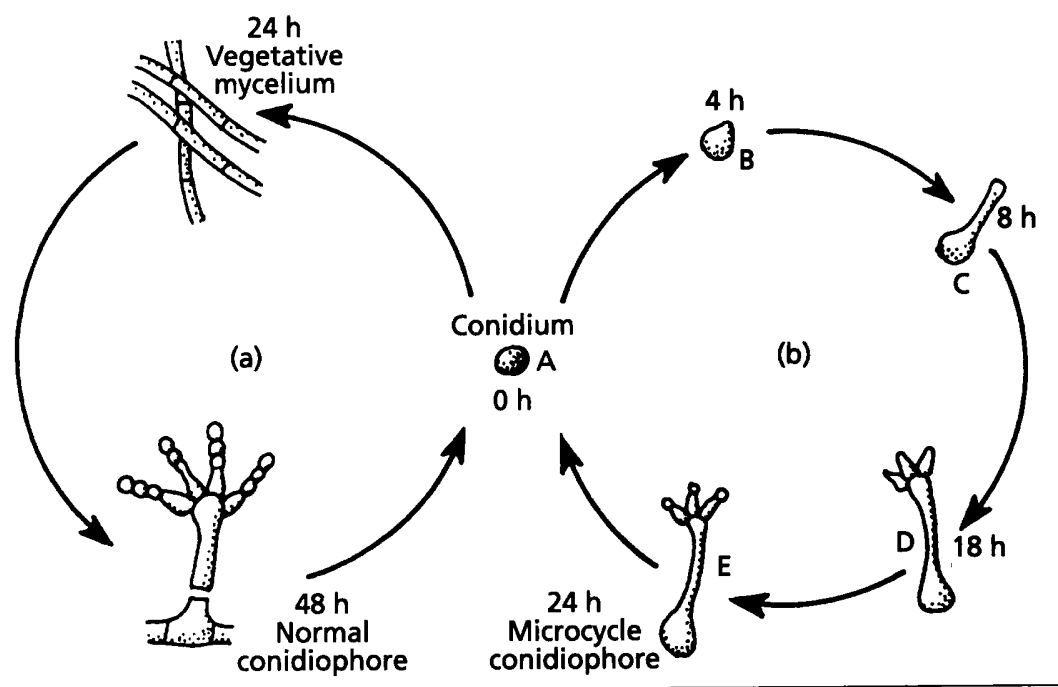

Fig. 1. Developmental pattern of A. flavus in (a) minimal medium and (b) D-GSS medium. A, conidium; B, conidial germination; C, germ-tube elongation; $D$, phialodogenesis; E, conidiogenesis. only $10 \%$ microcycle conidiophores were formed. Put at $0.5 \mathrm{mM}$ caused complete inhibition of MC. Increase in Put concentration also induced vegetative growth in a medium which otherwise supports MC (Fig. 2c). Sporadic normal conidiation was also observed on Put-amended medium but the conidiophores were immature without any vesicle induction (Table 2). Conversely, Spd had a weak inhibitory effect on $\mathrm{MC}$ as compared to Put, but resulted in a shift from MC to normal asexual differentiation (Table 2; Fig. 2d). Spd at 0.1 and $0.5 \mathrm{mM}$ caused about 20 and $30 \%$ inhibition of $\mathrm{MC}$, respectively. However, no MC could be detected in a medium fortified with $1 \mathrm{mM}$ Spd.

\section{Effect of PA synthesis inhibitors on MC}

Data on the effect of DFMO, DFMA, MGBG and CHA on $\mathrm{MC}$ in $A$. flavus are summarized in Figs 3-5. DFMO, an inhibitor of Put biosynthesis, induced a sharp decline in percentage $\mathrm{MC}$. Of the various concentrations of DFMO tested $(0 \cdot 01-5 \mathrm{mM})$, complete inhibition of MC was observed at $5 \mathrm{mM}$, and at $1 \mathrm{mM}$ only $2-3 \%$ conidia underwent MC (Fig. 3). DFMO also caused an increase in the percentage ungerminated conidia with a concomitant decrease in MC. Some conidia germinated at higher concentrations of DFMO (1 and $5 \mathrm{mM})$ to form thin germ tubes whose further growth and development were restricted even after the increase in the incubation period (Fig. 2e). By contrast, DFMA had a weak inhibitory effect on MC as compared to DFMO (Fig. 5) and it caused only $20-30 \%$ inhibition MC even at $5 \mathrm{mM}$ (Fig. 5). However, the microcycle conidiophores formed had a higher degree of intracellular vacuolation.

Spd biosynthesis inhibitors (MGBG and $\mathrm{CHA}$ ) at various concentrations tested $(0 \cdot 01-5 \mathrm{mM})$ produced successively greater inhibition of MC (Figs 4 and 5) with MGBG being more effective than CHA. MGBG at a low concentration $(0.5 \mathrm{mM})$ resulted in $50 \%$ inhibition of MC. However, complete inhibition of MC was observed only at $5 \mathrm{mM}$ MGBG (Fig. 4), and at $1 \mathrm{mM}$ around $20 \%$ MC was observed but the conidiophores were immature (Fig. 2h).
However, $\mathrm{CHA}$ was found to be less inhibitory, and $50 \%$ inhibition of MC was observed at $1 \mathrm{mM}$ (Fig. 5).

\section{Reversal of the inhibitory effects of PA inhibitors on MC by exogenously supplied PAs}

Spd at 0.01 and $0.1 \mathrm{mM}$ caused $85-90 \%$ restoration of inhibition of MC induced by $1 \mathrm{mM} \mathrm{DFMO}$ (Figs 3 and $2 \mathrm{~g}$ ). An increase in Spd concentration, however, was not that effective in reversing the inhibitory effect on MC, and a high degree of vegetative growth with sporadic normal conidiation was observed at $1 \mathrm{mM}$ Spd. Put, however, caused only partial reversion of $\mathrm{MC}$ as compared to the reversion frequencies obtained with Spd (Figs 3 and 2f). A similar reversion pattern by Put and Spd was recorded for MC inhibition induced by $5 \mathrm{mM}$ MGBG (Figs $2 \mathrm{i}$, $\mathrm{j}$, and 4). However, the microcycle conidiophores formed with $0.01 \mathrm{mM}$ Spd plus $5 \mathrm{mM}$ MGBG were immature (without vesicle induction) and complete maturity was attained only with $0.1 \mathrm{mM}$ Spd (Fig. 2j); sporadic normal conidiation was also observed in the latter treatment (Table 2).

\section{DISCUSSION}

Put, Spd and Spm are widely distributed in many organisms. In general, Spd is frequently the most abundant PA in fungi, Put being present in lower concentrations and Spm, if present, usually only in small amounts (Stevens \& Winther, 1979; Tabor \& Tabor, 1985). However, the present results show that three common PAs were present in $A$. flavus and Put was present in the highest concentration followed by Spd and Spm. Put, Spd and Spm have also been shown to be widely distributed in thermophilic moulds representing zygomycetes, ascomycetes and deuteromycetes along with the appreciable amounts of cadaverine (Singhania et al. 1991). Similar results were reported in Helminthosporium oryzae, Curvularia lunata, Pytbium aphanidermatum and Colletotrichum capsici (Bharti, 1995). Our results also imply that PA biosynthesis is active during germ-tube elongation and a 


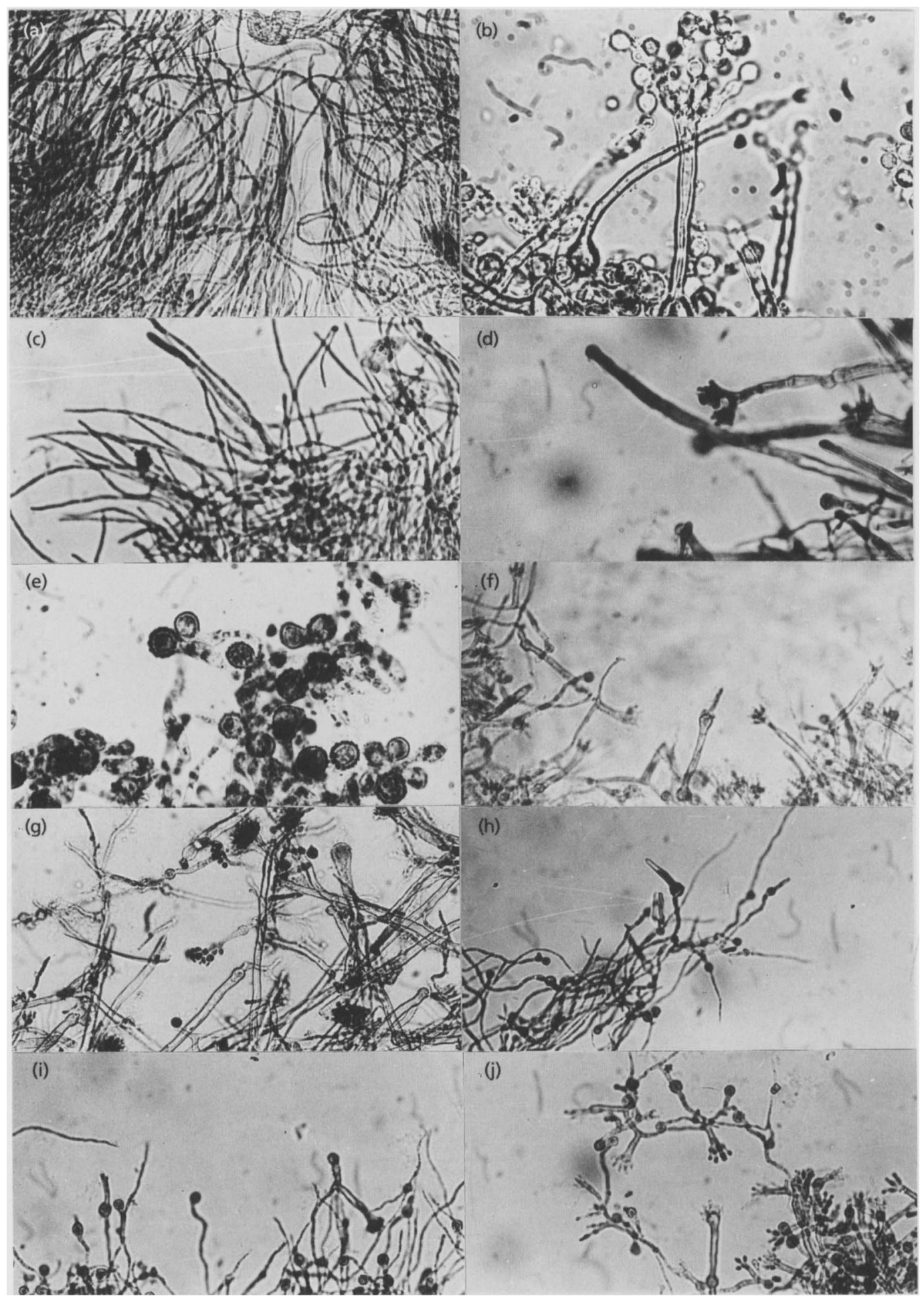

Fig. 2. Effect of $P A$ and/or PA biosynthesis inhibitors on $M C$ in $A$. flavus: (a) vegetative growth in minimal medium after $24 \mathrm{~h}$; (b) MC in D-GSS medium; (c) induction of vegetative growth by $0.5 \mathrm{mM}$ Put; (d) a shift from MC to normal conidiation by $0.5 \mathrm{mM}$ Spd; (e) suppression of germ-tube growth by $1 \mathrm{mM}$ DFMO; $(f)$ reversal of MC by supplementation of $0.1 \mathrm{mM}$ Put with $1 \mathrm{mM}$ DFMO; $(\mathrm{g})$ reversal of MC by $0.1 \mathrm{mM}$ Spd in $1 \mathrm{mM}$ DFMO; (h) suppression of MC by $1 \mathrm{mM}$ MGBG; (i) absence of MC reversion by $0.1 \mathrm{mM}$ Put in $5 \mathrm{mM}$ MGBG; (j) reversal of MC by $0.1 \mathrm{mM}$ Spd in $5 \mathrm{mM}$ MGBG. 
Table 2. Extent of asexual differentiation induced by exogenously applied PAs and/or PA inhibitors in MC in D-GSS medium

\begin{tabular}{|lcccccc|}
\hline $\begin{array}{l}\text { Treatment } \\
(\mathrm{mM})\end{array}$ & Put & Spd & MGBG & CHA & $\begin{array}{c}\text { MGBG (5 mM) } \\
+ \text { Put }\end{array}$ & $\begin{array}{c}\text { MGBG (5 mM) } \\
+ \text { Spd }\end{array}$ \\
\cline { 2 - 7 } & & & & & Extent of asexual differentiation* induced by: \\
\hline $0 \cdot 01$ & NT & NT & NT & NT & - & - \\
0.1 & ++ & ++++ & - & - & - & + \\
0.5 & + & ++++ & - & - & - & ++ \\
1.0 & + & +++ & - & - & NT & NT \\
5.0 & - & + & - & - & NT & NT \\
\hline
\end{tabular}

NT, Not tested.

$*++++$, Greater than $50 \% ;+++$, about $50 \% ;++$, less than $50 \%$, but greater than $10 \%$; + , less than $10 \% ;-$, not detected.

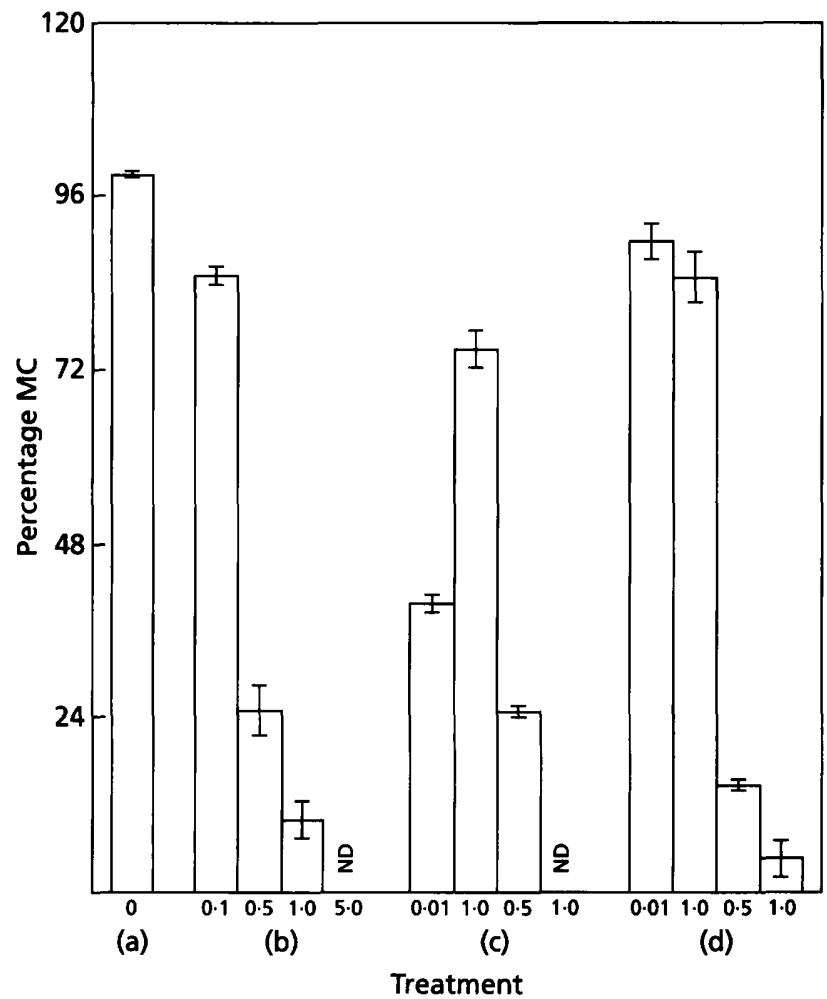

Fig. 3. Effect of DFMO singly and in combination with PAs (Put or Spd) on MC: (a) control; (b) 0.1-5.0 mM DFMO; (c) $1 \mathrm{mM}$ DFMO + 0.01-1.0 mM Put; (d) $1 \mathrm{mM}$ DFMO+0.01-1.0 mM Spd. ND, None detected.

high Put/Spd ratio is important for fungal spore germination and germ-tube elongation, whereas a low Put/Spd ratio supports spore differentiation to $\mathrm{MC}$. In other words, the drop in the Put/Spd ratio at the $18 \mathrm{~h}$ time point precedes with the onset of MC. PA biosynthesis has also been seen in early stages of germinating spores of wheat stem rust uredosporelings (Kim, 1971) and differentiation processes in Mucor rouxii (Calvo-Mendez et al.,

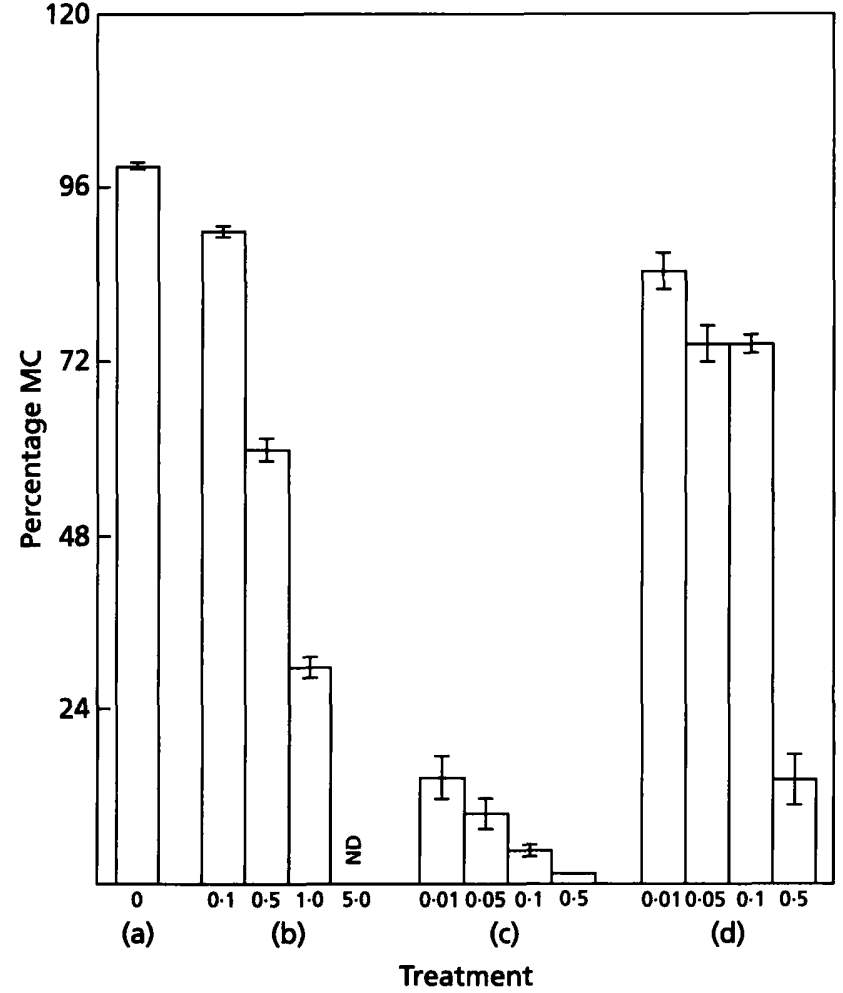

Fig. 4. Effect of MGBG singly and in combination with PAs (Put or Spd) on MC: (a) control; (b) 0.1-5.0 mM MGBG; (c) $5 \mathrm{mM}$ MGBG + 0.01-0.5 mM Put; (d) $5 \mathrm{mM} \mathrm{MGBG} \mathrm{+} \mathrm{0.01-0.5} \mathrm{mM} \mathrm{Spd.}$ ND, None detected.

1987; Martinez-Pacheco et al., 1989), and specific inhibitors of PA biosynthesis such as DFMO and/or DFMA (Rajam et al., 1985, 1986, 1989; Birecka et al., 1986; Machatsckke et al., 1990) and D-arginine (Gaur et al., 1989) can restrict spore germination/germ-tube growth/ sporulation in a variety of fungi (Tabor \& Tabor, 1985; Rajam, 1993). The role of PAs in the germination and outgrowth as ascospores of Saccharomyces cerevisiae has 


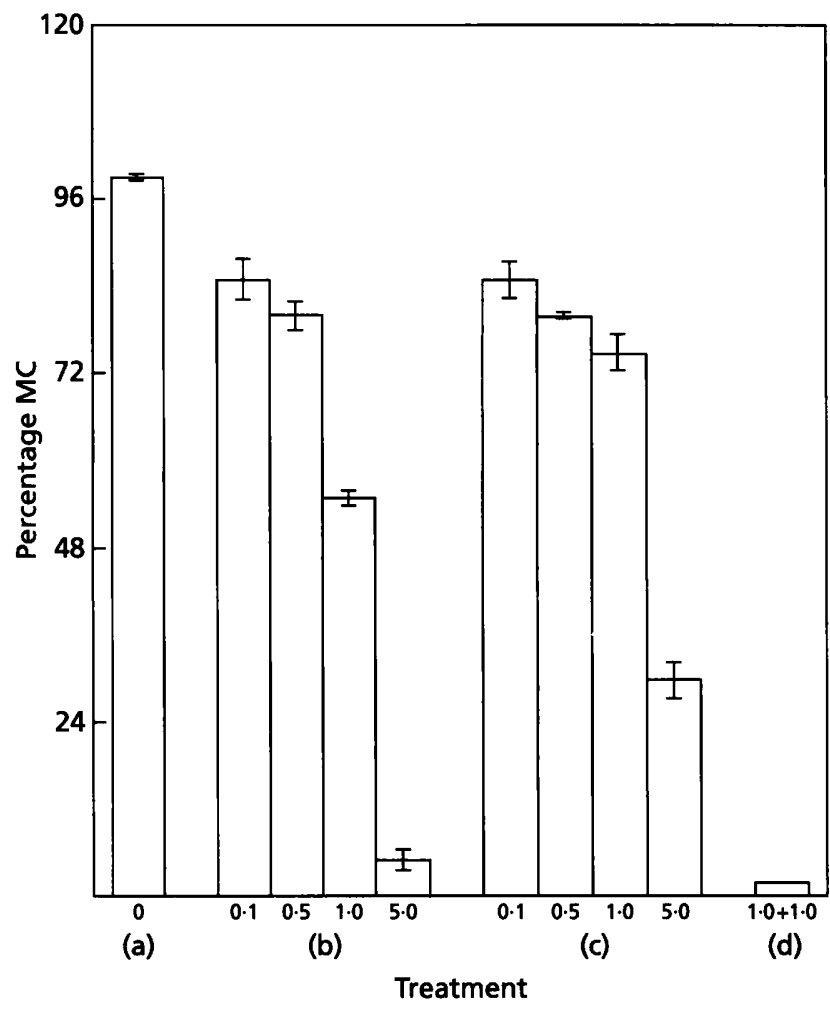

Fig. 5. Effect of CHA and DFMA singly and in combination with DFMO on MC: (a) control; (b) 0.1-5.0 mM CHA; (c) 0.1-5.0 mM DFMA; (d) 1 mM DFMA + 1 mM DFMO.

also been studied using $\alpha$-methylornithine and MGBG (Brawley \& Ferro, 1979). Germinating spores were found to be capable of initiating and progressing through the first cell cycle in the presence of PA inhibitors, but subsequent cellular growth was retarded but not completely arrested. Data further indicate that PA synthesis may be needed for meiosis and sporulation (Brawley \& Ferro, 1979). Besides this, the involvement of PAs, especially Spd for cell differentiation to embryoids (Feirer et al., 1984) or flower buds (Kaur-Sawhney et al., 1988) using cell suspensions or thin-layer cultures of carrot and tobacco, respectively, has been clearly demonstrated, and a low Put/Spd ratio was found to be essential for flower bud differentiation (Kaur-Sawhney et al., 1988).

The present findings clearly show that exogenous PAs and their biosynthetic inhibitors had a profound effect on MC. Put, while inhibiting MC, induced vegetative growth at a higher concentration $(1 \mathrm{mM})$ indicating its role in vegetative growth. In contrast, Spd had a weak inhibitory effect on MC but it resulted in a shift from MC to asexual differentiation. This indicates that higher cellular Spd levels then needed for $M C$ are required for asexual differentiation. Further, optimum rather than higher Spd levels, and an optimal Put/Spd ratio, seem to be important for MC. Calvo-Mendez et al. (1987) and Martinez-Pacheco et al. (1989) have shown that elevated levels of PAs precede all differentiation processes in $M$. rouxii, including yeast-mycelium transition. These variations were ac- companied by changes in ornithine decarboxylase (ODC, the key enzyme controlling PA biosynthesis in fungi) activity and inhibition of its activity by the reaction product analogue 1,4-diaminobutanone blocked spore germination at the transitions between the isodiametric and polarized growth stages, aerial mycelium formation, and the dimorphic yeast-mycelium transition (MartinezPacheco, 1989).

Our results showed a strong inhibition of $\mathrm{MC}$ by the Put synthesis inhibitor DFMO and this could be due to reduced levels of Spd as Put is the obligatory precursor of Spd formation or to defects in germ tube emergence. DFMA, however, was ineffective in suppressing MC, which implies that this fungus may possess only the ODC pathway for PA biogenesis like many other fungi (Walters, 1987; Rajam, 1993). Further, the weak effect of DFMA on MC may be due to the lack of the arginine decarboxylase (another key enzyme in PA synthesis) pathway in Aspergillus, or the lack of inhibitor uptake or a rapid metabolism of the inhibitor (Akhtar \& Minocha, 1989; Rajam et al., 1989; Bharti, 1995). The inhibition of MC by MGBG and CHA, which was associated with pronounced vegetative growth, was most likely due to the accumulation of inhibitor-induced Put as Put is not converted to Spd due to the blockage of adenosylmethionine decarboxylase and Spd synthase, respectively (Bharti \& Rajam, 1995). The induction of vegetative growth by MGBG or CHA could be because of availability of a minimal level of Spd and the high Put to Spd ratio as a result of inhibitor-induced Put accumulation (Bharti \& Rajam, 1995). This further supports the involvement of Put in mycelial growth. CHA was less effective on $\mathrm{MC}$ and it required much higher concentrations to get similar effects as MGBG; this may be because of poor uptake and rapid metabolism of the inhibitor, or because of insensitivity of the target enzyme (Akhtar \& Minocha, 1989; Rajam et al., 1989; Bharti, 1995). The partial/greater reversion of inhibitory effect of DFMO and MGBG on MC by Put and Spd, respectively, also indicates that PAs, particularly Spd, may be required for MC. The inhibitory effect of DFMO and other PA inhibitors on fungal growth and spore germination can be completely reversed by addition of Put or Spd to the medium (Rajam \& Galston, 1985; Rajam et al., 1989; Rajam, 1993).

In conclusion, these observations provide the first evidence that Spd is intricately involved in MC of $A$. flavus, and optimum, rather than higher, Spd levels and a low Put/Spd ratio seems to be important for MC. Further, these results may have possible utility to biotechnology and fermentation industries for mass inoculum production of industrially useful micro-organisms.

\section{ACKNOWLEDGEMENTS}

This investigation was supported in part by a grant to M. V.R. from the Department of Science and Technology (no. SP/ SO/A-23/92). The authors wish to thank Merrell Dow Research Institute, Cincinnati, USA, for the gift of DFMO and DFMA to M.V.R., Ms Bharti and Mr S. Bajaj for their help 
during the work, and Mr Rajiv Chawla for his expert secretarial assistance. N.K. is grateful to the University Grants Commission, New Delhi, for awarding a Junior Research Fellowship.

\section{REFERENCES}

Akhtar, J. K. \& Minocha, S. C. (1989). Polyamine biosynthetic enzymes and the effect of their inhibition on the growth of some phytopathogenic fungi. Plant Cell Pbysiol 30, 655-663.

Anderson, J. G. \& Smith, J. E. (1971a). The production of conidiophores and conidia by newly germinated conidia of Aspergillus niger (microcycle conidiation). J Gen Microbiol 69, 185-197.

Anderson, J. G. \& Smith, J. E. (1971b). Synchronous initiation and maturation of Aspergillus niger conidiophores in culture. Trans $\mathrm{Br}$ Mycol Soc 56, 9-29.

Bajaj, S. \& Rajam, M. V. (1995). Efficient plant regeneration from long-term callus cultures of rice by spermidine. Plant Cell Rep 14, 717-720.

Bharti (1995). Inbibition of fungal polyamine biosynthesis and control of leaf rust of wheat (Triticum aestivum L.): physiological and cytogenetical studies in the bost plant. PhD thesis, University of Delhi, India.

Bharti \& Rajam, M. V. (1995). Effects of the polyamine biosynthesis inhibitor difluoromethyl-ornithine on growth, polyamine levels, chromosome behaviour and polygenic traits in wheat (Triticum aestivum L.). Ann Bot 76, 297-301.

Birecka, H., Garraway, M. O., Baumann, R. I. \& McCann, P. P. (1986). Inhibition of ornithine decarboxylase and growth of the fungus Helminthosporium maydis. Plant Physiol 80, 798-800.

Brawley, J. V. \& Ferro, A. J. (1979). Polyamine biosynthesis during germination of yeast ascospores. J Bacteriol 140, 649-654.

Calvo-Mendez, C., Martinez-Pacheco, M. \& Ruiz-Herrera, J. (1987). Regulation of ornithine decarboxylase activity in Mucor bacilliformis and Mucor rouxii. Exp Mycol 11, 270-277.

Feirer, R., Mignon, G. \& Litvay, J. D. (1984). Arginine decarboxylase and polyamines required for embryogenesis in the wild carrot. Science 223, 1433-1435.

Gaur, S. C., Shekhawat, N. S. \& Arya, H. C. (1989). Growth and sporulation of millet leaf blast fungus Pyricularia penniseti: role of polyamines. Curr Sci 58, 198-200.

Kaur-Sawhney, R., Tiburcio, A. F. \& Galston, A. W. (1988). Spermidine and flower bud differentiation in thin-layer explants of tobacco. Planta 173, 282-284.

Khurana, N., Gupta, R., Kuhad, R. C. \& Saxena, R. K. (1992). Effect of protein synthesis and respiratory inhibitors on microcycle conidiation of Aspergillus tamarii. J Gen Appl Microbiol 38, 617-621.

Khurana, N., Gupta, R., Kuhad, R. C. \& Saxena, R. K. (1993). Light independent conidiation in Trichoderma spp. - a novel approach to microcycle conidiation. World J Microbiol \& Biotechnol 9, 353-356.
Kim, W. K. (1971). Folate and polyamine content of undiffrentiated and differentiated wheat stem rust uredosporelings. Can J Bot 49, 1119-1122.

Machatsckke, S., Kamrowski, C., Moerschbacher, B. M. \& Reisenger, H. J. (1990). Polyamine levels in stem rust infected wheat leaves and effects of alpha-difluoromethylornithine on fungal infection. Pbysiol Mol Plant Pbysiol 36, 451-459.

Maheshwari, R. (1991). Microcycle conidiation and its genetic basis in Neurospora crassa. J Gen Microbiol 137, 2103-2115.

Martinez-Pacheco, M., Rodriguez, G., Reyna, G., Calvo-Mendez, C. \& Ruiz-Herrera, J. (1989). Inhibition of the yeast mycelial transition and the phorogenesis of Mucorales by diaminobutanone. Arch Microbiol 151, 10-14.

Rajam, M. V. (1993). Polyamine biosynthesis inhibitors: new protectants against fungal plant diseases. Curr Sci 65, 461-469.

Rajam, M. V. \& Galston, A. W. (1985). The effects of some polyamine biosynthetic inhibitors on growth and morphology of phytopathogenic fungi. Plant Cell Pbysiol 26, 683-692.

Rajam, M. V., Weinstein, L. H. \& Galston, A. W. (1985). Prevention of a plant disease by specific inhibition of fungal polyamine biosynthesis. Proc Natl Acad Sci US A 82, 6874-6878.

Rajam, M. V., Weinstein, L. H. \& Galston, A. W. (1986). Kinetic studies on the control of the bean rust fungus (Uromyces phaseoli $\mathrm{L}$.) by an inhibitor of polyamine biosynthesis. Plant Pbysiol 82, 485-487.

Rajam, M. V., Weinstein, L. H. \& Galston, A. W. (1989). Inhibition of uredospore germination and germ tube growth by inhibitors of polyamine metabolism in Uromyces phaseoli L. Plant Cell Pbysiol 30, 37-41.

Saxena, R. K. (1976). Studies on the control of reproductive differentiation in Aspergillus nidulans under submerged culture conditions. $\mathrm{PhD}$ thesis, University of Delhi.

Saxena, R. K. \& Sinha, U. (1973). Conidiation of Aspergillus nidulans in submerged liquid culture. $J$ Gen Appl Microbiol 19, 141-146.

Saxena, R. K., Khurana, N., Kuhad, R. C. \& Gupta, R. (1992). DGlucose soluble starch, a novel medium for inducing microcycle conidiation in the genus Aspergillus. Mycol Res 96, 490-494.

Singhania, S., Satyanarayana, T. \& Rajam, M. V. (1991). Polyamines of thermophilic moulds: distribution and effect of polyamines biosynthesis inhibitors on growth. Mycol Res 95, 915-917.

Stevens, L. \& Winther, M. D. (1979). Spermine, spermidine and putrescine in fungal development. Adv Microb Pbysiol 19, 63-148.

Tabor, C. W. \& Tabor, H. (1985). Polyamines in microorganisms. Microbiol Rev 49, 81-99.

Walters, D. R. (1987). Polyamines: the Cinderellas of cell biology. Biologist 34, 73-76.

Received 30 May 1995; revised 9 October 1995; accepted 2 November 1995. 